\title{
Spinal cord injury and its association with blunt head trauma
}

This article was published in the following Dove Press journal:

International Journal of General Medicine

7 September 201 I

Number of times this article has been viewed

\author{
Wellingson S Paiva \\ Arthur MP Oliveira \\ Almir F Andrade \\ Robson LO Amorim \\ Leonardo JO Lourenço \\ Manoel J Teixeira \\ Division of Neurosurgery, \\ University of São Paulo, Brazi
}

Correspondence: Arthur Maynart Pereira Oliveira

Consolação Street N 2855 Ap 7I

Cerqueira Cesar, São Paulo, São Paulo,

Brazil, |416-00|

Tel +55 I| 30696329

Fax +55 II 30696000

Email ampo@infonet.com.br
Background: Severe and moderate head injury can cause misdiagnosis of a spinal cord injury, leading to devastating long-term consequences. The objective of this study is to identify risk factors involving spine trauma and moderate-to-severe brain injury.

Methods: A prospective study involving 1617 patients admitted in the emergency unit was carried out. Of these patients, 180 with moderate or severe head injury were enrolled. All patients were submitted to three-view spine series X-ray and thin cut axial CT scans for spine trauma investigations.

Results: 112 male patients and 78 female patients, whose ages ranged from 11 to 76 years (mean age, 34 years). The most common causes of brain trauma were pedestrians struck by motor vehicles $(31.1 \%)$, car crashes $(27.7 \%)$, and falls $(25 \%)$. Systemic lesions were present in 80 (44.4\%) patients and the most common were fractures, and lung and spleen injuries. 52.8\% had severe and $47.2 \%$ moderate head trauma. Fourteen patients $(7.8 \%)$ suffered spinal cord injury (12 in cervical spine, one in lumbar, and one thoracic spine). In elderly patients, the presence of associated lesions and Glasgow Coma Scale (GCS) $<9$ were statistically significant as risk factors $(P<0.05)$ for spine injury.

Conclusion: Spinal cord injury related to moderate and severe brain trauma usually affects the cervical spine. The incidence of spinal lesions and GCS $<9$ points were related to greater incidence of spinal cord injury.

Keywords: head injury, spine trauma, risk factors

\section{Introduction}

Although links between spine injuries, spinal cord injuries (SCI), or both, and traumatic brain injury (TBI) have been reported, the precise features of the injuries have not been described in detail. ${ }^{1,2}$ The reported incidence of cervical spine trauma after clinically significant head injury generally ranges from $4 \%$ to $8 \% .^{3-5}$ Acute spinal cord trauma in all spine segments occurs in $5 \%$ to $15 \%$ of patients with severe head injury. ${ }^{5,6}$ Although an increasing injury severity has been associated, as measured by the Glasgow Coma Scale (GCS), ${ }^{7}$ severe and moderate head injury may cause misdiagnosis of spinal cord injuries leading to devastating long-term consequences.

A head injury severe enough to cause coma transmits energy to the cervical spine; thus the prevalence of cervical spine injury among patients with traumatic coma is not insignificant. Few other risk factors in this patient population have been associated with a higher rate of spine injury. Virtually all of the literature fail to clearly define the basic radiologic investigation in unconscious patients. The objective of the present study is to identify and analyze the major risk factors involving spinal cord 
and moderate-to-severe brain injury at a Trauma Center in a Brazilian University Hospital.

\section{Material and methods}

This prospective study was designed from November 2004 to May 2006. One thousand, six hundred and seventeen patients were admitted into the emergency unit of the Hospital das Clínicas, Faculdade de Medicina, Universidade de Sao Paulo over this time period. Of these patients, 180 were prospectively assessed with moderate or severe head injury (postresuscitation GCS of 3-12). Only patients who had been managed in the Neurotrauma Intensive Care Unit were included in the study, that is, 112 male and 78 female patients, whose ages ranged from 11 to 76 years (mean age, 34 years). All patients were submitted to three-view spine series X-ray and thin axial CT scans in suspicious or poorly visualized areas.

The following traumatic head lesions were evaluated: skull vault fracture, skull base fracture, acute epidural hematoma, acute subdural hematoma, traumatic subarachnoid hemorrhage, intraventricular hemorrhage, and contusion hematoma. Potential risk factors and injuryrelated characteristics were documented including age, sex, mechanisms of trauma, location of injury, GCS score, and presence of neurological deficits or systemic lesions.

Patients with combined craniocervical injuries were managed in the intensive care unit after stabilization or surgical evacuation of an intracranial hematoma. Individuals with definitive or presumed SCI received methylpredinisolone, as indicated by the SCI protocol outlined in the National Acute Cord Injury Study II. Statistical significance was determined by Chi-square test with $P<0.05$.

\section{Results}

The most common causes of brain trauma were pedestrians struck by motor vehicles (31.1\%), car crashes (27.7\%), and falls $(25 \%)$. Systemic lesions were present in $80(44.4 \%)$ patients. Of the 14 patients with combined head and spine injuries, six were passengers, three were pedestrians struck by vehicles, two were assaulted, one was a motorcyclist who did not wear a helmet, and one suffered a fall.

Among 180 patients in the cohort (98 with an initial GCS score $<8$ and 82 cases with an initial GCS score of 9-12), 14 patients (7.8\%) suffered a spine injury. The most common findings in head CT scans among 14 patients with combined head and spine traumatic injury were diffuse swelling in seven patients, and subarachnoid hemorrhage in three.
SCI was observed in $7.8 \%$ of patients and 12 were located in the cervical spine, one in the lumbar and one in the thoracic spine. Age (older than 50), and presence of associated lesions and severe head injury were statistically significant $(P<0.05)$ as risk factors for spinal cord injury (Table 1$)$.

\section{Discussion}

Head injury and SCI are common occurrences in the neurosurgical practice. They account for an important proportion of care, disability, and ethical issues. They can be present in association with other conditions, and in these cases, the diagnosis of SCI may be difficult. Recognizing the risk factors for SCI associated with head injury is essential to prevent and reduce disability.

In the present study, no significant differences $(P>0.05)$ in gender distribution of TBI and SCI association were found. Male patients were predominant in the study $(81.1 \%$ males vs $18.9 \%$ females), whereas an almost equal distribution between male and female patients was found in the spine trauma group (7.5\% vs $8.9 \%)$.

Bucholz et $\mathrm{al}^{8}$ performed postmortem radiography and autopsy inspections in 100 consecutive victims of traffic accidents; they found a cervical spine injury rate of $24 \%$. In contrast, in patients with known head injury, the incidence of simultaneous spine injury has generally ranged from $4 \%$ to $8 \%$ which is similar to the figure found in the present study (7.8\%).

Age in our series was also an important factor. Age seems to have a great effect on the injury pattern in patients older than 50 years. Demetriades et $\mathrm{al}^{9}$ reported that age plays a great role in the anatomic distribution and severity of injuries and survival outcomes. Additionally the incidence of spinal injuries increased dramatically with age, and elderly patients were 21 times more likely to suffer spinal trauma than the pediatric population. ${ }^{9}$

In line with other analyses, ${ }^{10,11}$ we have shown that a road traffic crash is the most frequent mechanism of spinal injury, however no significant differences $(P>0.05)$ were verified during the study.

Table I Distribution of patients according risk factors to spinal cord injury associated with moderate and severe traumatic brain injury

\begin{tabular}{llll}
\hline Risk factors & $\%$ & $\%$ & $\boldsymbol{P}$ \\
\hline Sex & Male $=7.5 \%$ & Female $=8.9 \%$ & 0.48 \\
Age & $>50$ years $=5.3 \%$ & $>50$ years $=14.3 \%$ & $<0.05$ \\
Trauma mechanism & MVA & Low energy trauma & 0.33 \\
GCS & $3-8=13.7 \%$ & $9-12=1.2 \%$ & 0.021 \\
Systemic lesion & Yes $=12.5 \%$ & No $=4.0 \%$ & $<0.05$ \\
\hline
\end{tabular}

Abbreviations: GCS, Glasgow Coma Scale; MVA, motor vehicle accident. 
The GCS seems to play an important role in TBI and SCI association. Hills and Deanne ${ }^{5}$ report that patients with clinically significant head injuries were at greater risk of cervical spine injuries than those without head trauma (4.5\% vs $1.1 \%$, significant in the Chi-square analysis). Patients with GCS scores of 8 or less presented an even greater risk of spine injuries.

Our study has demonstrated that patients with TBI and associated injuries are at highest risk for associated spine injuries when patients presented with fractures, spleen, or lung injury. Martin et $\mathrm{al}^{12}$ concluded that chest injuries showed a more consistent relationship with spinal injury; $11.4 \%$ $(8.7 \%-14.1 \%)$ of patients with spine fracture/dislocation also suffered chest trauma, with a relative risk of $2.30(95 \%$ confidence intervals [CI]: 1.77-2.99; $P<0.001$ ); however, there was no increase in the relative risk of either fracture/ dislocation without cord injury (relative risk: 1.37 [95\% CI: $0.93-2.02 ; P=0.11]$ ), or with cord injury (relative risk: 0.49 [95\% CI: $0.12-2.00 ; P=0.31]$ ) associated with the presence of abdominal injury. Limb lesions were not associated with either type or injury. ${ }^{12}$

In our study, the cervical spine affected $86 \%$ of spine injury associated with TBI. Davis et $\mathrm{al}^{13}$ studied fatal craniospinal injuries and found a marked tendency for the spinal cord to be damaged with cervical segment involvement. Lida et $\mathrm{al}^{14}$ showed that in 188 patients with cervical spine and/or spinal cord injury, brain damage was frequently associated with cervical injury. Those patients with upper cervical injury are at greater risk of suffering from skull base fractures and severe intracranial hematomas.

\section{Conclusion}

Spinal cord injury related to moderate and severe trauma usually affects the cervical spine. The incidence of spinal lesions in this group of patients in our institution was $7.8 \%$. Patients older than 50 years, with systemic lesions, and those with an initial GCS score of 8 or less are at higher risk for concomitant spine injury.

\section{Disclosure}

No funds have been received to support this work. No benefits in any form have been or will be received from a commercial party related directly or indirectly to the subject of this manuscript.

\section{References}

1. Beachulis B, Long W, Hynes G, Johnson M. Clinical Indication For cervical spine Radiographs in the Traumatized patient. Am J Surg. 1987; 153:473-478.

2. Harris P. Associated injuries in traumatic paraplegia and tetraplegia. Parapledia. 1967;5:215-220.

3. Holly LT, Kelly DF, Cournelis GJ, Blinman T, McArthur DL, Cryer HG. Cervical Spine trauma associated with moderate and severe head injury: incidence, risk factors and injury characteristcs. J Neurosurg. 2002; 96 (3 Suppl):285-291.

4. Frye G, Wolfe T, Knopp R, et al. Intracranial hemorrhage as a predictor of occult cervical-spine fracture. Ann Emerg Med. 1994;23: 797-801.

5. Hills MM, Deanne SA. Head injury and facial injury: is there an increased risk of cervical spine injury? J Trauma. 1993;34: 549-554.

6. Michael DB, Guyot DR, Darmody WR. Coincidence of head and cervical spine injury. J Neurotrauma. 1989;6:177-189.

7. Teasdale G, Jennett B. Assessment of coma and impaired consciousness: a practical scale. Lancet. 1974;6:177-189.

8. Bucholz RW, BurkheAD WZ, Graham W, et al. Occult cervical spine injuries in fatal traffic accidents. J Trauma. 1979;19:768-791.

9. Demetriades D, Murray J, Martin M, Velmahos G, et al. Pedestrians injured by automobiles: relationship of age to injury type and severyty. Am Coll Surg. 2004;199:382-387.

10. Hedley MN, Zabramski JM, Browner CM, et al. Pediatric spinal trauma. Review of 122 cases of spinal cord and vertebral column injuries. J Neurosurg. 1998;68:18-24.

11. Osenbach RK, Menezes AH. Pediatric spinal cord and vertebral column injury. Neurosurg. 1992;30:385-390.

12. Martin BW, Dykes E, Leckey FE. Patterns and risks in spinal trauma. Arch Dis Child. 2004;89:860-865.

13. Davis D, Bolman H, Walker E, Fisher R, et al. The pathological findings in fatal craniospinal injuries. J Neurosurg. 1971;34:603-613.

14. Lida H, Tachibana S, Kitahara T, et al. Association of head trauma with cervical spine injury, spinal cord injury or both. J Trauma. 1999;46: $450-452$.
International Journal of General Medicine

\section{Publish your work in this journal}

The International Journal of General Medicine is an international, peer-reviewed open-access journal that focuses on general and internal medicine, pathogenesis, epidemiology, diagnosis, monitoring and treatment protocols. The journal is characterized by the rapid reporting of reviews, original research and clinical studies across all disease areas.

\section{Dovepress}

A key focus is the elucidation of disease processes and management protocols resulting in improved outcomes for the patient.The manuscript management system is completely online and includes a very quick and fair peer-review system. Visit http://www.dovepress.com/ testimonials.php to read real quotes from published authors. 